Interpersonal attraction: The role of agreement and topic interest. Journal of Personality \& Social Psychology, 1968, 9. 340-346.

CLORE, G. L., \& BALDRIDGE, B. The behavior of item weights in attitude-attraction research. Journal of Experimental Social Psychology, 1970, 6, 177-186.

FISHBEIN, M., \& HUNTER, R. Summation versus balance in attitude organization and change. Journal of Abnormal \& Social Psy chology, 1964, 69, 505-510.
KIESLER, C. A., COLLINS, B, E., \& MILLER, N. Attitude change. New York: Wiley, 1969 .

NOTES

1. Support for this hypothesis has been replicated in a separate study focusing on a different issue.

2. This result has also been replicated.

\section{The relationship between punishment and unavoidability in eliminating avoidance behavior in humans*}

\author{
WAYNE A. MAXWELL, FRANK D. MILLER, and PHILIP A. MEYER \\ University of South Dakota, Vermillion, S. Dak. 57069
}

Recent research has indicated that elimination of a persistent avoidance response (AR) is facilitated by an unavoidability procedure, wherein the noxious stimulus (NS) is presented on all trials whether an AR occurs or not, but it is facilitated even more by punishment wherein the NS is presented only following an $\mathrm{AR}$. The unavoidability and punishment procedures were conceptualized as extremes on a continuum designated as the percentage of nonresponses followed by the NS. Thus, four groups $(N=13)$ of undergraduates received the NS following each AR made during the elimination phase, but they differed with respect to the percentage of nonresponses followed by the NS: $0 \%$ (punishment), 25\%, 75\%, and 100\% (unavoidability). Several measures of performance during the elimination phase indicated significantly greater persistence as the percentage variable increased. These results were taken as supporting the original conceptualization.

A number of studies (cf. Beecroft, 1967, pp. 66-67) have indicated the generally high resistance to extinction of an avoidance response (AR). In an effort to facilitate elimination of the persistent AR, Meyer (1970) found that human Ss stopped avoiding significantly faster with an unavoidability procedure than with normal extinction. The unavoidability procedure consisted of presenting the

*This article is based on a thesis by the first author, which was supervised by the second and third authors and submitted to the Graduate School of the University of South Dakota in partial fulfillment of the requirements for the $M A$ degree.

tRequests for reprints should be sent to Philip A. Meyer, Department of Psychology, University of South Dakota, Vermillion, S. Dak. 57069 noxious stimulus (NS) on each trial regardless of whether $S$ made an $A R$ or not, whereas normal extinction consisted of omitting the NS on each trial, again regardless of $S$ 's behavior. In the same study, however, Ss punished for making an AR (i.e., presented the NS only when they made an AR) stopped responding even faster than Ss given the unavoidability procedure.

The present study was concerned with the difference between the unavoidability and the punishment procedures. While in both these procedures the NS is presented following an AR, they differ with respect to whether or not the NS is programmed to follow a nonresponse (i.e., when an AR does not occur). Specifically, the punishment procedure represents a condition in which a nonresponse is never followed by the NS, whereas in the unavoidability procedure, a nonresponse is always followed by the NS. These two procedures may be viewed as extremes on a continuum designated as the percentage of nonresponses followed by the NS (punishment $=0 \%$ unavoidability $=100 \%$ ). In order to determine further the usefulness of this interpretation, the present study varied the percentage of nonresponses followed by the NS in four groups: $0 \%, 25 \%, 75 \%$, and $100 \%$. Consistent with the methodology of Meyer (1970), human Ss received an air blast behind their right ear as the NS and learned a button depression as the AR. APPARATUS

The apparatus consisted of a response button mounted on the right arm of a dental chair with a headrest, a pressurized-air delivery system with a $1 / 8$-in. metal nozzle, a $71 / 2-W$ white light mounted on a wall in front of the S's head, relay switches, Hunter Interval Timers, and a Hunter Klockounter. Additional equipment included an earplug, an ace bandage which was tied around S's forehead and the headrest, and a mirror which allowed $\mathrm{E}$ to observe $\mathrm{S}$ continuously. PROCEDURE

The procedure generally followed that of Meyer (1970). Each $S$ was seated and instructed that an air blast would occasionally be directed behind his right ear; this air blast was described as annoying but not painful. $S$ was informed that there was a simple response he could make to control what happened in the experiment. The $S$ was also given the option of discontinuing the experiment at any time he wished. Following these instructions, the nozzle was positioned so that the air blast, which served as the NS, struck $S$ on the mastoid portion of the temporal bone, $1 / 2$ in. behind its juncture with the pinna.

All Ss received 40 training trials, wherein a depression of the response button during the 2 -sec signal light (i.e., an AR) terminated the signal and prevented the scheduled occurrence of a 4-sec NS at $40 \mathrm{psi}$. Any response during the NS was ineffective. The intertrial interval was 5 sec. As in Meyer (1970), Ss were prompted if they had not made three consecutive ARs by" Trial 10 and again, if needed, by Trial 20. Nineteen Ss required one prompt; an additional five required the second prompt.

Following training, an elimination phase occurred for which Ss were assigned randomly to one of four groups $(N=13)$. Except as noted below, the method of trial presentation was as in training. For all 
Table 1

Group Mean Number of ARs and Mean Trials to a Criterion of Eight Consecutive Nonresponses (TC8) During the Elimination Phase

\begin{tabular}{lrrrr}
\multirow{2}{*}{$\begin{array}{c}\text { Performance } \\
\text { Measure }\end{array}$} & \multicolumn{4}{c}{ Groups } \\
\cline { 2 - 5 } & \multicolumn{1}{c}{$\mathrm{P}$} & $\mathrm{NR25}$ & $\mathrm{NR75}$ & $\mathrm{U}$ \\
\hline ARs & 4.15 & 9.85 & 21.77 & $\mathbf{2 4 . 8 5}$ \\
TC8 & $\mathbf{1 3 . 3 8}$ & 36.92 & 53.46 & 51.15 \\
Efficiency (\%) & 97.92 & $\mathbf{7 3 . 9 2}$ & 50.69 & 44.08 \\
\hline
\end{tabular}

groups, an AR no longer prevented the NS but still terminated the signal. These groups differed with respect to the percêntage of nonresponses (i.e., failures to make an $A R$ ) which were contingently followed by the NS. Group $U$ received an unavoidability procedure in which $100 \%$ of the nonresponses were followed by the NS. At the other extreme, Group P never received an NS following a nonresponse $(0 \%)$, while being punished with the NS for making an AR. Groups NR25 and NR75 received NS presentations following $25 \%$ and $75 \%$ of the nonresponses, respectively. For Group NR25, the NS followed every fourth nonresponse; for Group NR75, the NS followed the last three of every four nonresponses. These schedules were more irregular than they appear, because interspersed among the nonresponse trials could be trials on which $S$ made an AR. All Ss were run to an extinction criterion of eight consecutive failures to respond or 50 trials, whichever occurred first. Response latencies were recorded to the nearest $0.01 \mathrm{sec}$.

\section{SUBJECTS}

The Ss were 52 men selected from an introductory psychology course at the University of South Dakota. Eight additional Ss were eliminated: one because of apparatus failure, three because of failure to reach the training criterion, two because of prior knowledge of the experiment, and two because they declined to finish.

RESULTS

During training, all Ss reached the criterion by Trial 30. Furthermore, there were no significant group differences in the number of ARs over the last 20 training trials, $F(3,48)=1.61, p>.10$.

Mean number of ARs and mean trials to an eight consecutive nonresponses criterion (TC8) during the elimination phase are presented in Table 1 for each group. A TC8 score of 58 was given Ss failing to reach the criterion. As shown in Table 1 , the several elimination procedures were differentially effective in suppressing avoidance behavior. With respect to the number of ARs measure, Group P suppressed responding most rapidly and Group $U$ suppressed least rapidly, with Groups NR25 and NR75 performing serially between these two extremes. An analysis of variance was performed on the number of ARs and yielded significant group effects, $\mathrm{F}(3,48)=17.33, \mathrm{p}<.001$. Post hoc comparisons, using the Tukey $B$ procedure, substantiated the significant differences between all group pairs except Groups NR75 and $\mathrm{U}(\mathrm{p}<.01)$. In the case of the TC8 measure, the results were essentially the same. Analysis revealed significant group effects, $\quad F(3,48)=33.74$, $\mathrm{p}<.001$. Also, the Tukey B procedure again substantiated the significant differences between all group pairs except Groups NR75 and U $(p<.01)$.

Also given in Table 1 is the mean efficiency score for each group. This score is the mean percentage of nonresponses for all trials during the elimination phase which followed a S's first nonresponse and is used here in the same manner as employed by Meyer (1970). The' efficiency measure represents the "suddenness" with which a $\mathbf{S}$ stops responding. A high efficiency score indicates one-trial the efficiency score was zero. As with the other measures, significant differences occurred, indicating greater efficiency in suppressing responding as the percentage of nonresponses followed by NS presentations decreased, $F(3,48)=18.67, p<.001$. Again, post hoc comparisons with the Tukey B procedure substantiated significant differences in efficiency between all group pairs except Groups NR75 and $U(p<.05)$.

The group mean latency of ARs in both training and the elimination phase was calculated for Groups NR25, NR75, and U (latencies were not calculated for Group P, since there were so few responses for this group). The mean avoidance latencies in training were $.47, .44$, and .55 sec for Groups NR25, NR75, and $U$, respectively, and during the elimination phase the mean latencies were $.56, .55$, and $.67 \mathrm{sec}$ respectively. While no significant group differences in avoidance latency occurred $(p>.10)$, analysis indicated significant overall increases in latencies from training to the elimination phase, $\mathrm{F}(1,36)=30.16, \mathrm{p}<.001$, and no significant interaction effect, $\mathrm{F}<1$. cessation. If a $S$ responded on all trials,

\section{DISCUSSION}

The present results clearly show that the degree of persistence of avoidance behavior in humans is a direct function of the percentage of nonresponses followed by NS presentations during an elimination phase in which all ARs are also followed by the NS. This monotonic trend was evidenced by several measures of performance during the elimination phase (i.e., number of ARs and trials to criterion). Furthermore, the "suddenness" with which Ss stopped responding during the elimination phase, as measured by the efficiency score, increased as the percentage of nonresponses followed by the NS decreased. In addition, when ARs did occur during the elimination phase, they tended to have longer latencies than in training. With specific reference to the unavoidability and punishment groups, the above findings are in complete agreement with earlier work (Meyer, 1970) which included these identical groups.

The suggestion has been made that the basis for the greater efficiency of punishment over the unavoidability procedure in eliminating avoidance behavior is the nature of the contingencies which follow nonresponding. The direct relationship obtained in the present study serves as confirmation and support for this interpretation. Moreover, this interpretation emphasizes the instrumental contingencies involved in the avoidance situation rather than the classical contingencies. Previously, theoretical emphasis of the instrumental contingencies which follow the AR has been made (e.g., Bolles, 1970; Herrnstein, 1969); however, the current emphasis is on the contingencies of nonresponding, the alternative to making an AR. An ambiguity in the present formulation arises since presentation of the NS may be taken equally well as nonreinforcement or punishment. Thus, alternative interpretations of the present resuits which focus on either the nonreinforcing or punishing aspects of the various experimental conditions are applicable to the present findings.

\section{REFERENCES}

BEECROFT, R. S. Emotional conditioning Psychonomic Monograph Supplements, 1967, 2(4, Whole. No. 20).

BOLLES, R. C. Species-specific defense reactions and avoidance learning. Psychological Review, 1970, 77, 32-48.

HERRNSTEIN, R. J. Method and theory in the study of avoidance. Psychological Review, 1969, 76, 49-69.

MEYER, P. A. The role of an unavoidability procedure in eliminating avoidance behavior with humans. Journal of Experimental Psychology, 1970,86 . 337-340. 